\title{
Shear-driven instability in zirconium at high pressure and temperature and its relationship to phase-boundary behaviors
}

\author{
M. K. Jacobsen, ${ }^{1,}{ }^{*}$ N. Velisavljevic, ${ }^{1}$ Y. Kono, ${ }^{2}$ C. Park, ${ }^{2}$ and C. Kenney-Benson ${ }^{2}$ \\ ${ }^{1}$ Shock and Detonation Physics (M-9), Los Alamos National Laboratory, Los Alamos, New Mexico 87545, USA \\ ${ }^{2}$ HPCAT, Geophysical Laboratory, Carnegie Institution of Washington, Argonne, Illinois 60439, USA
}

(Received 18 December 2016; revised manuscript received 13 March 2017; published 5 April 2017)

\begin{abstract}
Evidence in support of a shear driven anomaly in zirconium at elevated temperatures and pressures has been determined through the combined use of ultrasonic, diffractive, and radiographic techniques. Implications that these have on the phase diagram are explored through thermoacoustic parameters associated with the elasticity and thermal characteristics. In particular, our results illustrate a deviating phase boundary between the $\alpha$ and $\omega$ phases, referred to as a kink, at elevated temperatures and pressures. Further, pair distribution studies of this material at more extreme temperatures and pressures illustrate the scale on which diffusion takes place in this material. Possible interpretation of these can be made through inspection of shear-driven anomalies in other systems.
\end{abstract}

DOI: 10.1103/PhysRevB.95.134101

\section{INTRODUCTION}

Zirconium (Zr), a member of the group IV-B metals, is a fascinating material and has elicited much study due to industrial and military applications. This is primarily due to both the low neutron cross section and high degree of corrosion resistance. In addition, zirconium is used in refractory applications and is prolific in mineral species. A wide array of work exists studying the equation of state with temperature [1-11]. However, there is a decided absence of other physical property measurements (e.g., elasticity, thermal conduction, etc.), where such information can enable further advances.

Under ambient conditions, $\mathrm{Zr}$ crystallizes in a hexagonal close-packed structure (a.k.a. $\alpha$ phase), but pressure is known [1] to induce a transformation to a more open hexagonal structure ( $\omega$ phase). This is of particular importance, as the room temperature-pressure structure of transition metals tends to follow the canonical hcp $\rightarrow$ bcc $\rightarrow$ hcp $\rightarrow$ fcc structural sequence with increasing atomic number [12] (i.e., increasing electron number). Comparatively, pressure has been demonstrated to induce a similar phase sequence in several of the transition metals. In particular, it is well known that zirconium makes the transformation from hcp $\rightarrow$ bcc (or $\beta$ phase) at elevated temperature and pressure as would be expected. However, the intermediate $\omega$ phase has presented issues and possible explanations regarding its existence have been presented in many reports [13-17].

The phase diagram of $\mathrm{Zr}$ has been investigated in several works, primarily involving $\mathrm{x}$-ray diffraction studies. For example, Zhao et al. [18] probes the phase diagram up to $17 \mathrm{GPa}$ and $1000 \mathrm{~K}$, resulting in x-ray determined equations of state for all $(\alpha, \omega$, and $\beta)$ phases known to exist in this

\footnotetext{
*mjacobsen@lanl.gov

Published by the American Physical Society under the terms of the Creative Commons Attribution 4.0 International license. Further distribution of this work must maintain attribution to the author(s) and the published article's title, journal citation, and DOI.
}

range. Similar work has been published by Zhang et al. [7] wherein they explore the high pressure and temperature phase boundaries using x-ray diffraction. Other similar studies have been reported, but inspection of typical equation of state forms can illustrate the limitations of such a study. One of the most commonly applied is the Birch-Murnaghan equation of state [19], which takes the form,

$$
\begin{aligned}
P= & \frac{3}{2} B_{0}\left[\left(\frac{V_{0}}{V}\right)^{7 / 3}-\left(\frac{V_{0}}{V}\right)^{5 / 3}\right] \\
& \times\left\{1-\frac{3}{4}\left(4-B_{0}^{\prime}\right)\left[\left(\frac{V_{0}}{V}\right)^{3 / 2}-1\right]\right\},
\end{aligned}
$$

where $P$ is the pressure, $B_{0}$ is the isothermal bulk modulus, $V_{0}$ is the temperature dependent volume at zero pressure, $V$ is the volume at $P$ and $T$ conditions, and $B_{0}^{\prime}$ is the first pressure derivative of the bulk modulus. With this equation, the bulk modulus and pressure evolution can be determined, along with ambient pressure volumes. However, complete elasticity information contains both the bulk and shear moduli along with their pressure and temperature dependencies.

In contrast to x-ray only studies, full elasticity can be obtained from ultrasonic techniques (UT) [20,21], which has probed minerals subject to high pressure conditions. This technique, however, has only recently been made widely available for studies in other areas (e.g., metals [22]). Although such experiments can be difficult to perform, they provide the opportunity to simultaneously determine bulk and shear moduli and their pressure derivatives, in addition to other possible parameters. Due to the nature of UT, they can be more sensitive to bulk material changes compared to just measurements with $\mathrm{x}$-ray diffraction on the same material. For instance, previous high-pressure UT experiments on $\mathrm{Zr}$ [23] allowed identification of the coexistence region for the $\alpha$ to $\omega$ transformation from 4 to $6 \mathrm{GPa}$ along with corresponding elasticity values across the two phases.

Beyond just the physical property information, there are several aspects of the phase diagram that are lacking explanation or detailed investigation. The phase boundaries for zirconium are known to be highly dependent on sample 
purity, with a higher composition of interstitial impurities resulting in a dramatic increase in the $\alpha \rightarrow \omega$ phase transition pressure [24,25]. However, the actual phase boundary is quite difficult to pin down, as evidenced by many works $[5,7,17,18,23-29]$, where the prime difficulty is wide $\alpha-\omega$ coexistence. Other interesting features exhibited include high pressure-temperature amorphous phases [7,30] and phase boundaries that exhibit possible kinks [7,27] (i.e., change from positive to negative $\mathrm{dT} / \mathrm{dP}$ phase boundary slope). This report presents high pressure-temperature studies on zirconium in the range up to $7 \mathrm{GPa}$ and $1400 \mathrm{~K}$ using UT and simultaneous $\mathrm{X}$-ray diffraction and radiography. Results from this work clearly support the kink in the $\alpha-\omega$ boundary and relates it directly to phonon mode competition, likely related to soft phonon modes in the crystal lattice. The kink itself appears in the phase boundary at approximately $600 \mathrm{~K}$ and $4 \mathrm{GPa}$. Additionally, evidence from this work suggests a reconsideration of the possible amorphous phase at elevated pressure and temperature, which is likely related to the phonon mode competition as has been found in another system with similar behavior [31].

\section{EXPERIMENTAL METHODS}

\section{A. Ultrasonic and $x$-ray studies}

High P-T elasticity experiments have been done using beamline 16-BM-B (HPCAT, Advanced Photon Source) at Argonne National Laboratory, with technical details presented in a previous report [32]. Samples were prepared from the highest purity material available, as used in other works [25,33]. External diameters of $1.5 \mathrm{~mm}$ and thicknesses of approximately $0.8 \mathrm{~mm}$ were were polished to better than $1 \mu \mathrm{m}$ finish. Each sample was loaded into a sample assembly, described in a previous report [34], designed for ultrasonic measurements in a VX-3 Paris-Edinburgh press. Piezoelectric transducers (Boston Piezooptics, dual mode $Y$-cut $10^{\circ}$ ) were mounted on the backside of the top anvil, with sound waves creating reflections at each boundary between sample cell components due to acoustic impedance mismatch [34].

\section{B. Pair distribution function studies}

Pair distribution studies, also accomplished in the VX-3 Paris-Edinburgh Press at 16-BM-B, are performed by taking diffraction patterns at varying angles covering the whole accessible range of the instrumentation. For our studies, this involved patterns obtained at two-theta angles of 4, 5, 7, 9, $11,14,18,22$, and 28 degrees. Subsequent to the collection, the energy range from $40-65 \mathrm{keV}$ from each pattern has been processed to remove crystalline peaks and combined/converted to evaluate the structure factor measured. All processing of the data is done using software available at the beam line and developed specifically for this purpose.

\section{Data processing and pressure/temperature control}

Radiographic images of the sample are then used to determine sample thickness (1) with a Prosilica GE1380H model camera, where contrast is used to determine sample boundaries. Sound velocities are determined from contrast
TABLE I. Experimental details for ultrasonic investigation of $\mathrm{Zr}$ with pressure and temperature. This table lists the maximum pressure and temperature reached in each experiment, along with the pressure marker used in each experiment. For the pressure markers, $\mathrm{Au}$ and $\mathrm{NaCl}$ mixture or $\mathrm{MgO}$ have been used, both in the form of a sleeve directly surrounding the sample. The annotation $P$ after the temperature indicates that the path used was to increase to a set temperature and then increase pressure (i.e., isothermal compression) to the maximum pressure value listed.

\begin{tabular}{lccc}
\hline \hline Exp. \# & Max. $P(\mathrm{GPa})$ & Max. $T(\mathrm{~K})$ & P. Marker \\
\hline 1 & 6.59 & 300 & $\mathrm{Au} / \mathrm{NaCl}$ \\
2 & 4.41 & 1050 & $\mathrm{Au} / \mathrm{NaCl}$ \\
3 & 5.23 & $523^{P}$ & $\mathrm{MgO}$ \\
4 & 5.14 & $523^{P}$ & $\mathrm{MgO}$ \\
5 & 4.19 & 1300 & $\mathrm{Au} / \mathrm{NaCl}$ \\
6 & 5.79 & 1400 & $\mathrm{MgO}$ \\
7 & 2 & 1000 & $\mathrm{MgO}$ \\
\hline \hline
\end{tabular}

determined radiographic lengths and pulse-echo determined round trip travel times as $v_{x}=2 l / t_{x}$, with $x$ being either $p$ for compressional waves or $s$ for shear waves. Sample densities $(\rho)$ and unit cell volumes $(V)$ have been determined from energy dispersive $\mathrm{x}$-ray diffraction patterns, using MDI's JADE software. Internal pressure has been determined using a sleeve around the sample made either from a mixture of $\mathrm{Au}$ and $\mathrm{NaCl}$ or pure $\mathrm{MgO}$, with unit cell parameters used with established equations of state [35,36]. Longitudinal (L) and shear $(\mathrm{G})$ moduli at each high pressure and temperature point have been calculated from the corresponding wave velocities and densities, as $L=\rho v_{p}^{2}$ and $G=\rho v_{s}^{2}$, with $p$ and $s$ as defined above. The adiabatic bulk modulus is determined from the longitudinal and shear moduli as $B=L-4 G / 3$. The refined $\mathrm{X}$-ray sample volumes have been analyzed with the Birch-Murnaghan equation of state [Eq. (1)] to determine an independent isothermal bulk modulus $\left(B_{0}\right)$ and pressure derivative $\left(B_{0}^{\prime}\right)$. These fits were done only for experimental temperatures where three or more data points existed for a fit. Details of the individual experiments is given in Table I. Temperature determination was performed by either type $\mathrm{K}$ (Alumel-Chromel) or type $\mathrm{C}(\mathrm{W} / 5 \% \mathrm{Re}-\mathrm{W} / 26 \% \mathrm{Re})$ thermocouple. Based on this, the estimated temperature accuracy of these measurements is $\pm 10 \mathrm{~K}$, with pressure effects on the thermocouples disregarded.

\section{Calculated parameters}

In this paper, there are an assortment of computations made for various values. It has previously been demonstrated that ultrasonic measurements can be used to determine elastic Deybe temperatures [37,38], Grüneisen parameters [39], and ultimately to convert adiabatic bulk moduli to isothermal [40]. Standard relations between the sound velocities and the Poisson's ratio have also been used. Results from these determinations are shown in Fig. 2. In particular, the average/mean sound velocity, which is defined as

$$
v_{m}=\left(\frac{v_{p}^{-3}+2 v_{s}^{-3}}{3}\right)^{-1 / 3} .
$$


From this mean sound velocity, the elastic Debye temperature is determined from

$$
\Theta_{D, \text { elastic }}=\frac{h}{k_{b}}\left(\frac{3 N_{A} \rho}{4 \pi a}\right)^{1 / 3} v_{m}
$$

with $a$ being the atomic weight, $h$ being the Planck constant, and $\rho$ being the density. The acoustic Grüneisen parameter is obtained directly from the sound velocities through

$$
\gamma=\frac{3}{2}\left(\frac{3\left(v_{p} / v_{s}\right)^{2}-4}{\left(v_{p} / v_{s}\right)^{2}+2}\right) .
$$

The elastic Debye temperature is then used to compute the lattice thermal conductivity as

$$
\lambda_{\text {lat }}=8\left(\frac{k_{b}}{h}\right)^{3} \frac{M V^{1 / 3} \Theta_{D}^{3}}{\gamma^{2} T}
$$

with $V$ being the unit cell volume, $M$ the formula mass per unit cell, $T$ being the temperature, and $\gamma$ being the Grüneisen parameter. Finally, the isothermal bulk modulus is determined as

$$
B_{T 0}=\frac{B_{S 0}}{1+\alpha \gamma T} .
$$

Young's modulus and the Poisson ratio can also be determined from the acquired data, through relations from the bulk and shear moduli. In the computation of the isothermal bulk modulus, the thermal expansion coefficient $(\alpha)$ used is from reference data reported by Goldak et al. [41].

It should be noted here that the acoustic Grüneisen parameter and subsequent parameters determined through its use are subject to some assumptions that may affect the interpretation of the results. The derivation of the functional form presented and the assumptions associated with it can be found elsewhere [42]. Of fundamental importance here is the assumption of Debye-like behavior, which may or may not be a safe assumption to make for zirconium. This parameter should be recognized as fundamentally different from the thermodynamic Grüneisen parameter and will likely not agree with thermodynamic values.

\section{RESULTS}

Ultrasonic data and x-ray diffraction patterns, along with $\mathrm{x}$-ray radiographs, have been processed into sound velocities, densities, and pressures as described in the Methods section. Specific $P-T$ points and a color mapping of the resulting longitudinal modulus are laid out, with an overlay of reported phase boundaries, in Fig. 1. The shear and adiabatic bulk modulus are also shown in this figure.

In these results, there are three principle findings to note. The first is the distinct softening of the shear modulus around $550 \mathrm{~K}$, as seen in Fig. 1(b). In particular, this softening extends from approximately $1 \mathrm{GPa}$ out to the proposed boundary with the $\omega$ phase.

The second feature is the distinct weakening of all moduli near the phase boundary between $\alpha$ and $\beta$ phases, located below $3 \mathrm{GPa}$. The shear modulus further weakens with increasing pressure and temperature. While such behavior may be expected, due to softening with increasing temperature, this feature shows a degree of extreme localization suggesting
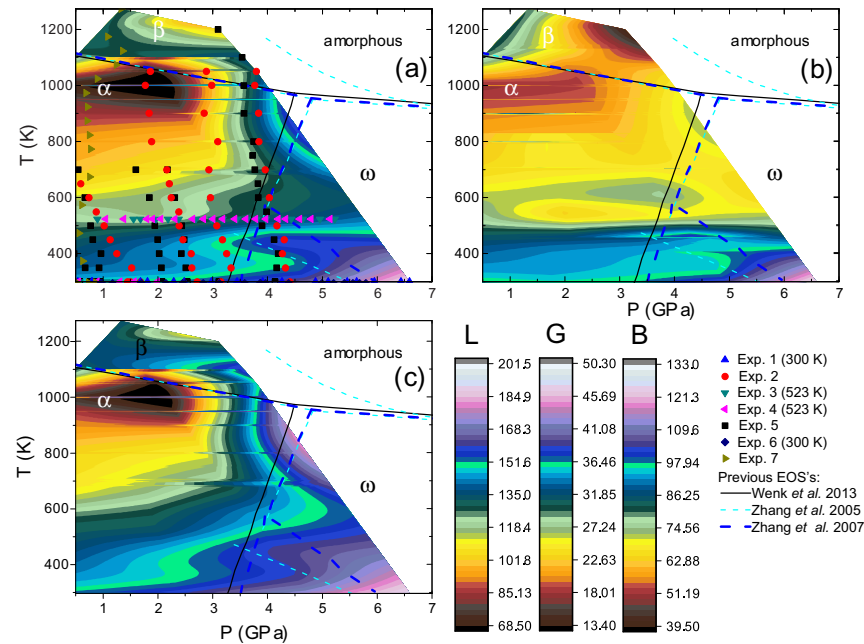

FIG. 1. Contour plots of the longitudinal modulus (a), shear modulus (b), and adiabatic bulk modulus (c), all in GPa, are shown over the $P-T$ range explored through this work. Overlaid on these plots are proposed phase boundaries from work by Wenk [17] and Zhang [7,27] and colleagues. The actual data points measured are plotted in (a) along with the other information. In these plots, the main points of interest are clearly seen, as described in the text. The left and right branches of the results from Zhang et al.'s paper [7] have been determined as follows. The left branch is directly from the phase boundaries reported (Figure 4 of their paper). The right branch is extracted from the experimental data points they show in this figure. From the latter of these, there is clearly a deviation in the phase boundary in contrast to the dashed phase boundary shown.

that it is likely associated with something phenomenologically different.

Beyond just the elastic properties obtained, the x-ray results have also been used to confirm the proposed phase transformation boundaries with applied pressure. Examples across each of the boundaries are exhibited in Figure 3. These
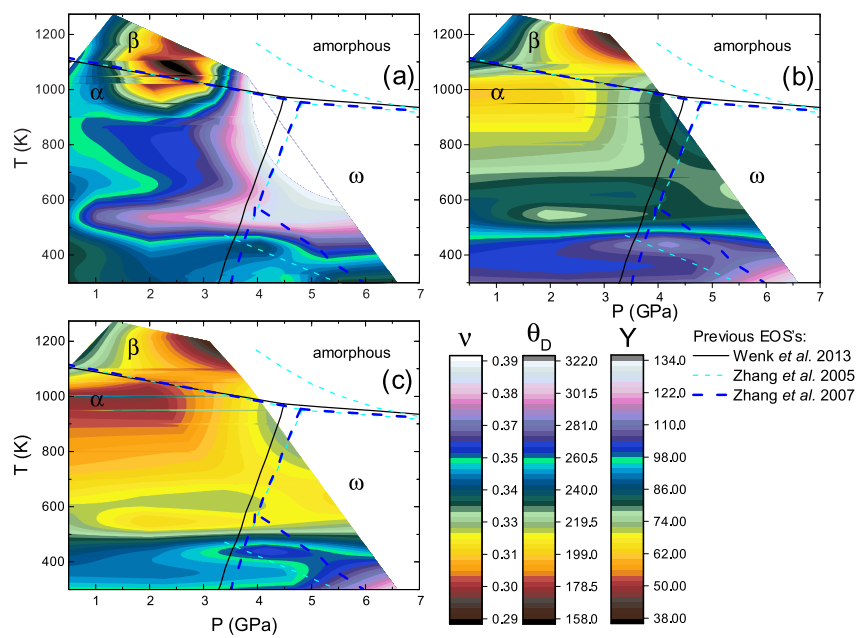

FIG. 2. Other parameters of zirconium have also been determined. In particular, Poisson's ratio (a), the elastic Debye temperature in K (b), and the Young's Modulus in GPa (c). Overlaid on the contour plot are proposed phase boundaries from work by Wenk [17] and Zhang [7,27] and colleagues. 


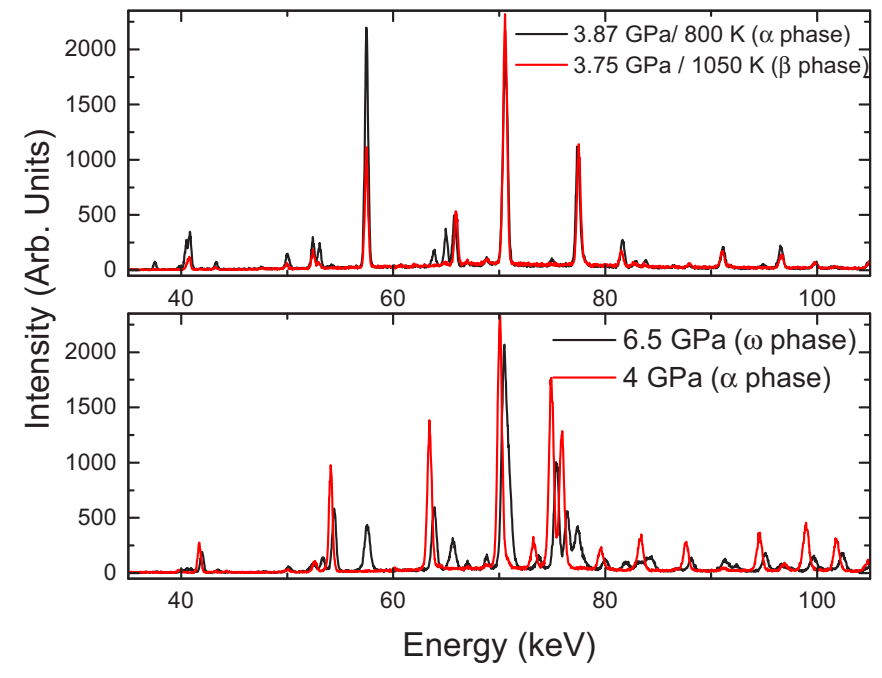

FIG. 3. To more closely inspect the x-ray evidence of the phase transformations, the obtained patterns can be shown across the $\alpha-\omega$ (ambient temperature) and $\alpha-\beta$ boundary. In both cases, there are clear changes demonstrating that the crystalline structure is altered, coincident with the changes in the elastic properties measured.

x-ray results have also been fit using the Birch-Murnaghan equation of state [Eq. (1)], with these results compiled in the Supplemental Material [43]. Similarly, all computed and measured elastic parameters have been compiled in the Supplemental Material [43].

\section{DISCUSSION}

\section{A. Comparison with other works}

Ambient temperature results have been compared with the ambient temperature results of Liu et al. [23], as shown in Fig. 4 along with bulk modulus values at both 300 and $523 \mathrm{~K}$. At ambient temperature $(300 \mathrm{~K})$, phase transition initiation appears around $4.9 \mathrm{GPa}$ and completes near to $6 \mathrm{GPa}$, as determined from $\mathrm{x}$-ray and elastic data collectively. Raising the temperature to $523 \mathrm{~K}$ shifts these pressures to $3 \mathrm{GPa}$ and
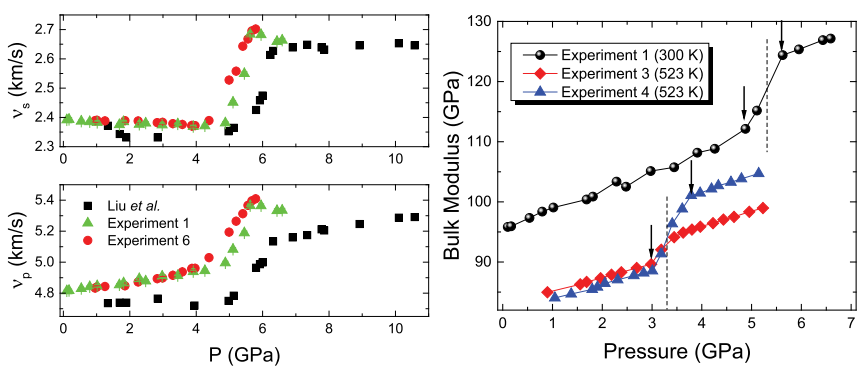

FIG. 4. Sound velocities for ambient temperature zirconium with pressure are shown for compressional $\left(v_{p}\right)$ and shear $\left(v_{s}\right)$ waves (left). In this, only data from solely ambient temperature experiments is included and compared with Liu et al. [23] data for the same temperature/pressure range. Errors for this plot are represented by the size of the symbol. The bulk modulus (right) can also be used to determine phase transition regions, where our results show a clear shift to lower pressure with increasing temperature.
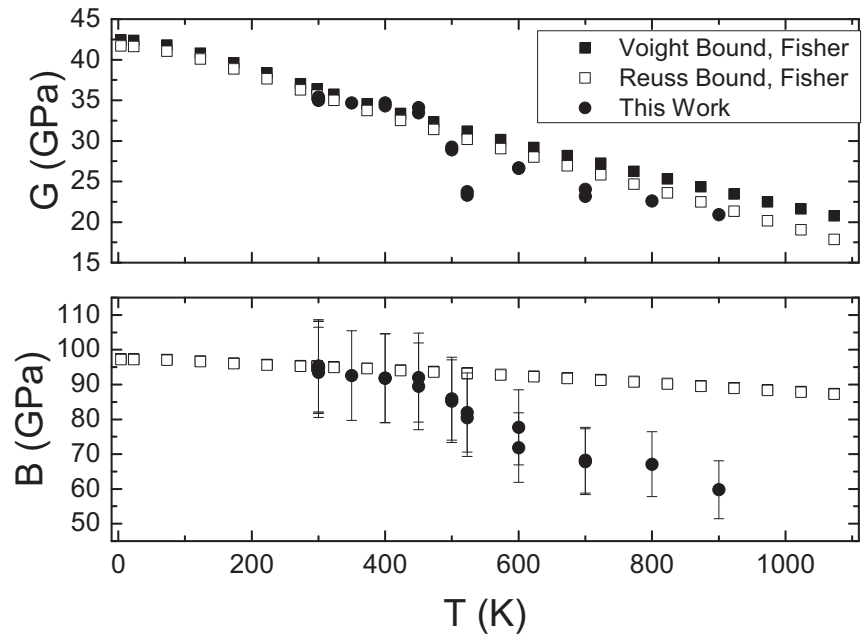

FIG. 5. Comparison of the elastic properties determined from this work have been compared with single crystal elastic moduli determined from Fisher et al. [44]. Possible reasons for the deviations are discussed in the text.

completion to near $4 \mathrm{GPa}$. Deviations between the results here and those from Liu et al. are most likely due to errors in the pressure determination, as both setups use $\mathrm{x}$-ray surrogates to determine internal pressure in the setup.

These results have also been compared with the previously reported temperature dependence of the single crystal elastic constants, presented by Fisher et al. [44], and are shown in Fig. 5. These results present both the Voight and Reuss bounds from the single crystal elastic constants in comparison with the least-squares fitted ambient pressure values from this work. As can be seen, aside from the rather dramatic drop in the shear modulus around $500 \mathrm{~K}$, the single crystal bounds match fairly well with the results here. In contrast, the bulk modulus agrees up to approximately the same region, from where it drops off more dramatically. Reasons for this discrepancy could include bulk material versus single crystal results, pressure/temperature induced effects, and sample related effects. For example, the samples used in this work are of extremely high purity, as discussed in the Methods section. In contrast, Fisher [44] references previous work to discuss sample preparation [45]. In this, they clearly mention that approximately $10 \mathrm{ppm}$ (by weight) of hydrogen was present in two of the three crystals used for velocity measurement. As it is known that impurity presence can dramatically effect the resulting sample properties, it is possible that such an influence was present. However, it should also be considered that the ambient pressure values used here are extrapolations of the high pressure data, as no measurements were made at ambient pressure during these experiments. Therefore, it is entirely possible that the influence of high pressure phenomena explored in this work have effected these results and provided the resulting difference. Despite the apparent agreement between the shear results measured here and Fisher, there is a clear change in slope across the discontinuous phenomena near $500 \mathrm{~K}$. Further work is planned to explore this difference in greater depth. 


\section{B. Phase boundary kink and shear softening}

Continuing the analysis with the $\alpha-\omega$ phase boundary. Upon inspection of the previously proposed phase boundaries [7,27] between the $\alpha$ and $\omega$ phases, there is the suggestion that temperatures between 300 and approximately $550 \mathrm{~K}$ result in a negative pressure slope for the transition pressure as a function of temperature. This trend is obtained from the results presented by Zhang et al. [7], where the straight lines appearing in the overlays are from their plotted phase boundaries. In contrast, the data plotted in their graph clearly indicates that the $\alpha-\omega$ boundary shifts to higher pressure below $550 \mathrm{~K}$, as is shown in Fig. 4 of their report. Overall, this is consistent with the measured coexistence region reported both here and in the work from Liu et al. [23]. The deviation, or "kinked," boundary has been estimated from their plot and included here in Figs. 1 and 2.

This trend reverses above this temperature to the $\beta$ boundary. While no particular mechanism for the change in phase boundary slopes has been suggested, the authors of one work [27] stated that the shift is due to "dynamic" effects without elaboration. External support for the kinked phase boundary comes from reversion studies from $\omega$ to $\alpha$, which have shown that the temperature required to stimulate reversion to the ambient phase must be in excess of $470 \mathrm{~K}$ [15]. Further theoretical support for the kinked phase boundary has also recently been provided by Yeddu et al. [46], whose report shows clearly a kinked phase boundary from simulational results. From our results, we propose that shear softening and the phase boundary kink are coupled in nature. With regard to the sharp decrease in the shear modulus at elevated temperature [Fig. 1(b)] and the associated kink in the phase boundary, this is likely a shear instability associated with phonon mode competition.

A similar situation has been previously reported for coesite [31], where soft phonon modes were found to alter the shear stability of the crystal structure. Previous work from Heiming et al. [47] studied zirconium's phonon modes and dispersion. Results from their work show that two low energy phonon modes $\left[\mathrm{L} \frac{2}{3}(1,1,1)\right.$ and $\left.\mathrm{T}_{1} \frac{1}{2}(1,1,0)\right]$ provide the displacements necessary for the martensitic transitions to $\alpha$ (T mode) and $\omega$ (L mode) structures. Further, Heiming proceeds to explain that the displacement of the $\mathrm{L}$ mode phonon and the $\mathrm{T} 1$ $\mathrm{N}$-point phonon are identical to the transverse $\mathrm{T}$ mode phonons discussed. As such, they draw the conclusion that, as they say, “...all phonons which achieve the displacements necessary for the two martensitic transitions lie in the same phonon branch with $[\xi \xi 2 \xi]$ propagation and transverse polarization" [47]. Due to this, it is possible for these two low-lying, overdamped phonons, connected through a similarly low-lying and overdamped phonon band, to transform or affect each other. Ultimately, this give support to the idea that the $\mathrm{L}$ and $\mathrm{T}$ modes could affect the opposite polarization and its strength characteristics.

\section{Possible amorphization}

In addition to these results, our results also provide some evidence suggesting that the previous reports regarding amorphization of zirconium at elevated pressures and temperatures should be re-evaluated. Similar to previous studies [7,30],
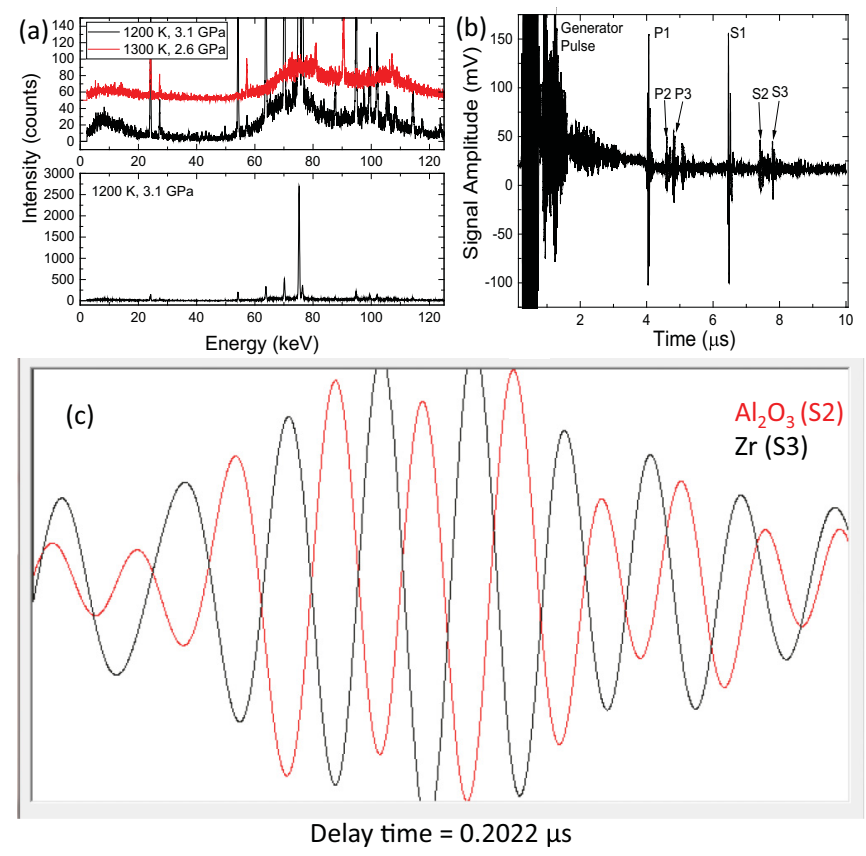

FIG. 6. Heating of the zirconium sample into the beta phase region initially results in clear $\beta$ phase x-ray patterns (a) (detector angle $=7.5^{\circ}$ ), but further heating results in loss of the pattern features and the emphasis of an amorphous appearing background signal. Additionally, the top panel of (a) shows an zoomed version of the lower pattern in red. As will be presented in more detail later, this background pattern is present from temperatures around $900 \mathrm{~K}$ and is present well into the $\beta$ phase. To verify that this signal is not from a liquid, concurrent ultrasonic pulse-echo patterns (b) show a shear wave reflection from the sample, providing a clear indication of a solid sample. To better illustrate this, the overlapped pattern (c) is presented with indicators showing which waveform is from which reflection.

results from this work have shown loss of diffraction at a single diffraction angle, as illustrated in Fig. 6(a). As verification that our results do not observe a melting phenomenon, pulse-echo ultrasound results have been analyzed to observe a shear reflection from the sample, indicating clearly a solid sample. This is further supported by radiographic images of the sample that show no melting, as flow of the liquid would be observed out of the sample area. It should be noted that, in contrast to other works [7,30], all samples were taken from $\alpha$ to $\beta$ and never from $\omega$ to $\beta$. As such, it is interesting to note that the amorphouslike behavior was observed over the whole of the $\alpha-\beta$ boundary investigated to the lowest pressure measurable $(\approx 0.5 \mathrm{GPa})$.

However, the amorphous claim was suggested incorrect by Hattori et al. [30], wherein they used angle-dispersive x-ray diffraction to suggest the cause was more likely rapid grain growth related to lattice instability in the $\beta$ phase. As it is difficult to tell the difference from $x$-ray diffraction alone, we have performed PDF studies over the whole range of available angles, an example of which is shown in Fig. 7.

This result shows a striking similarity to the diffuse background measured and reported in Hattori's work [30], as shown in Fig. 8. In their work, Hattori et al. suggests that the results of the diffuse scattering are related to the lattice 


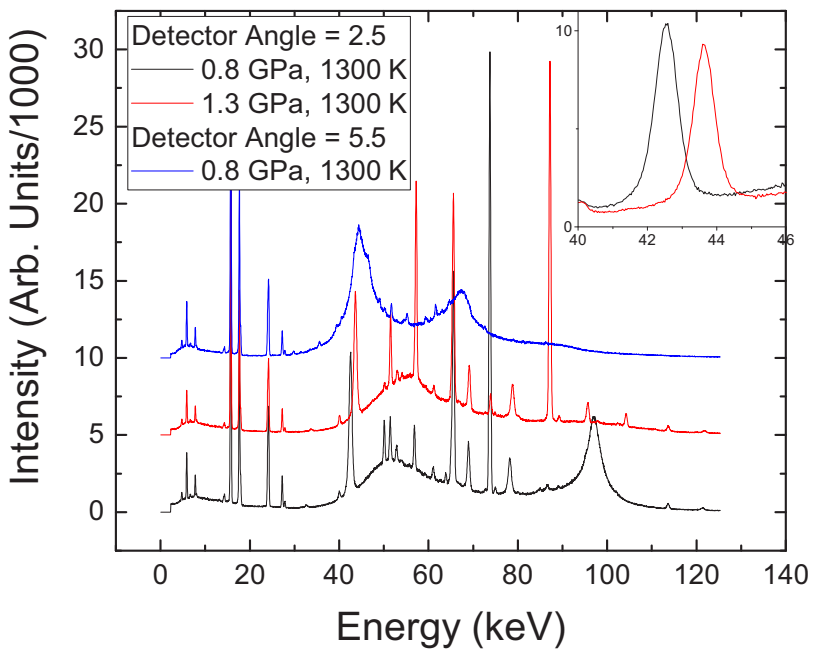

FIG. 7. Example results from the EDXRD patterns taken which show clearly the change in full width with increasing pressure, along with the crystalline peaks present in the patterns. The inset shows a close up of one particular peak around $42 \mathrm{keV}$. These show a full width of $0.77 \mathrm{keV}$ for the $0.8 \mathrm{GPa} / 1300 \mathrm{~K}$ pattern and $0.72 \mathrm{keV}$ for the $1.3 \mathrm{GPa} / 1300 \mathrm{~K}$ pattern. Fits of these peaks were performed using a Pearson-VII peak shape for both, with an error of $0.09 \mathrm{keV}$.

instability in the $\beta$ phase. Despite the striking similarity, the peak positions found from the structure factor results here are not at the regular intervals suggested from the diffuse scattering results.

The further conclusion of rapid grain growth can also be checked from these results. Upon inspection of the crystalline peaks, as shown in Fig. 7, the full width at half maximum (FWHM) shows a decrease over the same $P-T$ region. These

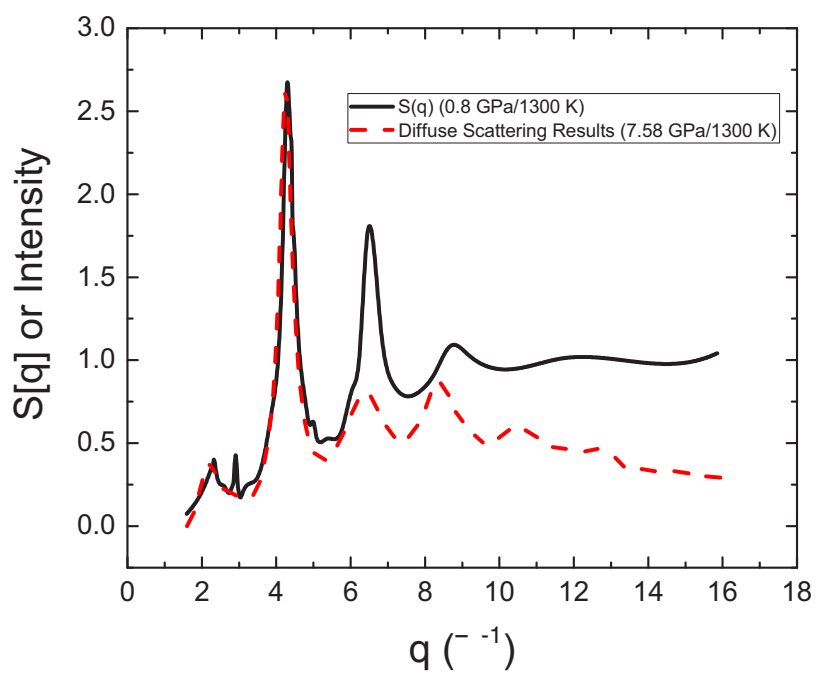

FIG. 8. Upon conversion/subtraction of the 40-65 keV regions of the energy dispersive x-ray diffraction patterns (EDXRD), the structure factor extracted shows a similar regularity to that observed by Hattori et al. [30] (red dashed curve). Although the conclusion posed by their work suggests that this is due to rapid anomalous crystal growth, we suggest that this could be explained by partial amorphization of the material as discussed in the text. show a full width of $0.77 \mathrm{keV}$ for the $0.8 \mathrm{GPa} / 1300 \mathrm{~K}$ pattern and $0.72 \mathrm{keV}$ for the $1.3 \mathrm{GPa} / 1300 \mathrm{~K}$ pattern. Fits of these peaks were performed using a Pearson-VII peak shape for both, with an error of $0.09 \mathrm{keV}$. Despite this, the error present in the values makes these two values overlap. Assuming that the decrease is real, explanation of this decrease can be accomplished with the Scherrer equation

$$
\tau=\frac{K \lambda}{\beta \cos (\theta)}
$$

where $\tau$ is the mean size of the crystallites, $K$ is a shape factor, $\lambda$ is the wavelength, $\theta$ is the Bragg angle, and $\beta$ is the full width at half maximum. In particular, the narrower the full width $(\beta)$ is, the larger the crystallite size is and vice versa. Therefore, the small decrease in FWHM would suggest a small degree of grain growth in the crystalline portion of the specimen. It is important to note that temperature was not changed during the compression phase of the EDXRD results shown. These results then indicate pressure-induced grain growth in the crystalline phase, as opposed to the temperature induced grain growth explored in Zhang [7] and Hattori's [30] works.

In contrast, comparison of the background peak shapes with the obviously crystalline ones illustrates how difficult it would be to find a single crystallite size that would explain the background features. The crystalline peaks all have similar peak shapes and, therefore, similar crystallite sizes could be determined from them. In the $0.8 \mathrm{GPa}$ pattern, the background peaks, which are located around $47 \mathrm{keV}$ and $95 \mathrm{keV}$ in the $0.8 \mathrm{GPa}$ pattern and around $50 \mathrm{keV}$ in the $1.3 \mathrm{GPa}$ pattern, have different shapes. Similar behavior is seen in other two-theta settings as well. As such, it seems reasonable to postulate that amorphization is a potential cause for the background. It should be noted that the technique used for this analysis, namely energy dispersive $\mathrm{x}$-ray diffraction, has a much larger instrumental broadening than angle dispersive diffraction does. To further explore the results discussed in this paragraph, further experimentation using angle dispersive diffraction is planned.

Similar to the pressure dependent growth, there is also a component of temperature dependent change. As is shown in Fig. 9, the increase in temperature shows a change in position and full width. This directly suggests a change in the cause of the background, but also shows that if this is ultimately determined to be amorphization, the amorphization extends in temperature at least down to $900 \mathrm{~K}$ and likely is related to shear softening related to the kink. Although there is clearly not enough support to claim that there is a partially amorphous phase near the $\alpha-\beta$ boundary, there are several conclusions that can be made from the data available. The fact that the broad background extends and is influenced by temperature down to at least $900 \mathrm{~K}$ suggests it is reasonable to pose that the background features are linked to the shear instability between the $\alpha-\omega$ boundary. Similarly, the unusual thermoacoustic properties near the $\alpha-\beta$ boundary are most likely associated with the background and, therefore, also associated with the shear instability. Finally, since the phenomena observed are similar to those found in silica [31], it would be a reasonable extension that some amorphization should be present. This must be moderated with the condition that complete amorphization does not appear possible for zirconium and 


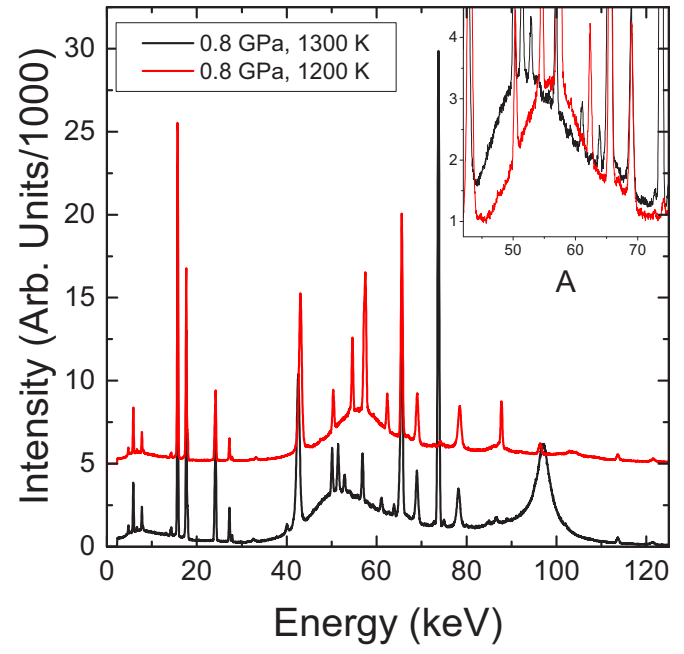

FIG. 9. Example results from the EDXRD patterns taken demonstrating changes in the background peaks with increasing temperature. In this, the black curve is at $0.8 \mathrm{GPa}$ and $1300 \mathrm{~K}$, whereas the red curve is $900 \mathrm{~K}$. As can be seen, there is a distinct shift in position and possible breadth as well with increase in temperature.

what is observed is at best partial amorphization, if it is amorphization.

\section{Hydrostaticity and kinetics effects}

As this work is all done in a Paris-Edinburgh press setup, the consideration of how hydrostaticity might affect these results is important. In particular, hydrostatic conditions are known to have a substantial effect on the transition pressure [15,24,25] and could potentially have an influence on application of these results. The determined start of the transition region $(4.9 \mathrm{GPa})$ is consistent with that previously reported for nonhydrostatically loaded $\mathrm{Zr}$ (4.6 GPa [25]). In contrast, the completion of the transition occurs at approximately $6 \mathrm{GPa}$, which is consistent with a hydrostatically loaded high purity specimen (6.4 GPa [25]). Thus, it is difficult from just the phase coexistence regions to determine the hydrostatic nature of the experiment. If one considers just the x-ray results, the conclusion is that these experiments are relatively hydrostatic (quasihydrostatic), as the transition is not detected by $\mathrm{x}$ ray until the upper end of the transition region. This latter supposition seems the most reasonable, as the results being compared with are all x-ray diffraction studies of zirconium. Further, the work of Liu et al. [23] shows a similar transition region for a study performed in a multianvil apparatus (X17B2, NSLS), which is known to provide relatively hydrostatic conditions [48].

It is also possible that the transition region could be influenced by kinetics, as has been demonstrated at ambient temperature previously [49]. From this work, it was determined that the extent of transformation between the two phases was strongly influenced by how rapidly the pressure was changed and by how much. The larger the change, the more complete the transformation. In contrast, the more rapid the change, the shorter the timescale required for the transformation to occur. As such, the relatively slow pressure changes and small step size in this work would induce a very slow and sluggish transition between the two phases. This would likely have an impact on the results through partial conversion between the two phases. However, its effect on the kink position and phase boundary remains uncertain and further work is required to elucidate this.

\section{CONCLUSIONS}

Combined ultrasonic and $\mathrm{x}$-ray studies of zirconium at elevated pressures and temperatures have been performed and have revealed several unusual phenomena at play. Results on the thermoacoustic properties of $\mathrm{Zr}$ have demonstrated a marked change in the shear modulus occurring around $523 \mathrm{~K}$ $\pm 10 \mathrm{~K}$ and extending from approximately $1 \mathrm{GPa}$ out to the previously indicated kink in the $\alpha-\omega$ phase boundary at nearly $4 \mathrm{GPa}$. Further, these results have illustrated an unusually localized softening of the moduli near the $\alpha-\beta$ phase boundary and the increasing Grüneisen parameter at both ambient and elevated temperatures. Additional information on the elastic Deybe temperature, isothermal bulk modulus, and Poisson's ratio have also been reported.

Overall, the first two of these unusual phenomena seem to be likely associated with phonon mode competition induced by temperature and pressure. Previous reports from Heiming et al. [47] have illustrated that two low energy phonon modes in the $\beta$ phase result in the necessary displacements to form the $\alpha$ and $\omega$ structures. We propose based on the results of this work that the competition between these two modes results in the dramatic shear softening around $523 \mathrm{~K}$ and the associated kink in the phase boundary. This conclusion is directly associated with the elasticity measured in the material through pulse-echo ultrasound probes. Further, some evidence suggesting a reinvestigation of previous reports regarding amorphization is needed. In particular, grain size analysis of energy dispersive diffraction results and structure factor analysis results suggest the possibility of an amorphous component of the high pressure, high temperature specimen.

\section{ACKNOWLEDGMENTS}

Los Alamos National Laboratory (LANL) is operated by LANS, LLC for the DOE-NNSA under Contract No. DE-AC52-06NA25396. The authors acknowledge funding support from LANL Science Campaigns 1 and 2. Portions of this work were performed at HPCAT (Sector 16), Advanced Photon Source (APS), Argonne National Laboratory. HPCAT operations are supported by DOE-NNSA under Award No. DE-NA0001974 and DOE-BES under Award No. DE-FG0299ER45775, with partial instrumentation funding by NSF. APS is supported by DOE-BES, under Contract No. DEAC02-06CH11357. Use of the Advanced Photon Source, an Office of Science User Facility operated for the US Department of Energy (DOE) Office of Science by Argonne National Laboratory, was supported by the US DOE under Contract No. DE-AC02-06CH11357. MKJ gratefully acknowledges the support of the U.S. Department of Energy through the LANL/LDRD Program and the G.T. Seaborg Institute for this work. The authors also thank Dr. Baosheng Li for the use of his pulse-echo overlap software for determination of transit times. 
[1] J. Jamieson, Science 140, 72 (1963).

[2] A. Jayaraman, W. Klement, and G. Kennedy, Phys. Rev. 131, 644 (1963).

[3] Y. K. Vohra, S. K. Sikka, and R. Chidambaram, J. Phys. F 9, 1771 (1979).

[4] I. Folkins, M. B. Walker, and Z. Y. Chen, Phys. Rev. B 44, 374 (1991).

[5] C. W. Greeff, Modell. Simul. Mater. Sci. Eng. 13, 1015 (2005).

[6] D. Errandonea, Y. Meng, M. Somayazulu, and D. Häusermann, Physica B 355, 116 (2005).

[7] J. Zhang, Y. Zhao, C. Pantea, J. Qian, L. L. Daemen, P. A. Rigg, R. S. Hixson, C. W. Greeff, G. T. Gray, Y. Yang, L. Wang, Y. Wang, and T. Uchida, J. Phys. Chem. Solids 66, 1213 (2005).

[8] I. Schnell and R. C. Albers, J. Phys.: Condens. Matter 18, 1483 (2006).

[9] E. K. Cerreta, J. P. Escobedo, P. A. Rigg, F. L. Addessio, T. Lookman, C. A. Bronkhorst, C. P. Trujillo, D. W. Brown, P. O. Dickerson, R. M. Dickerson, and G. T. Gray III, EPJ Web Conf. 26, 02013 (2012).

[10] P. S. Ghosh, a. Arya, R. Tewari, and G. Dey, J. Alloys Compd. 586, 693 (2014).

[11] H. Zong, T. Lookman, X. Ding, C. Nisoli, D. Brown, S. R. Niezgoda, and S. Jun, Acta Mater. 77, 191 (2014).

[12] R. S. Hixson, D. A. Boness, J. W. Shaner, and J. A. Moriarty, Phys. Rev. Lett. 62, 637 (1989).

[13] M. P. Usikov and V. A. Zilbershtein, Phys. Status Solidi A 19, 53 (1973).

[14] A. Rabinkin, M. Talianker, and O. Botstein, Acta Metall. 29, 691 (1981).

[15] S. K. Sikka, Y. K. Vohra, and R. Chidambaram, Prog. Mater. Sci. 27, 245 (1982).

[16] D. R. Trinkle, R. G. Hennig, S. G. Srinivasan, D. M. Hatch, M. D. Jones, H. T. Stokes, R. C. Albers, and J. W. Wilkins, Phys. Rev. Lett. 91, 025701 (2003).

[17] H.-R. Wenk, P. Kaercher, W. Kanitpanyacharoen, E. ZepedaAlarcon, and Y. Wang, Phys. Rev. Lett. 111, 195701 (2013).

[18] Y. Zhao, J. Zhang, C. Pantea, J. Qian, L. L. Daemen, P. A. Rigg, R. S. Hixson, G. T. Gray, Y. Yang, L. Wang, Y. Wang, and T. Uchida, Phys. Rev. B 71, 184119 (2005).

[19] F. Birch, Phys. Rev. 71, 809 (1947).

[20] B. Li, R. C. Liebermann, and D. J. Weidner, J. Geophys. Res. 106, 579 (2001).

[21] Y. Higo, Y. Kono, T. Inoue, and K.-i. Funakoshi, J. Synchrotron Radiat. 16, 762 (2009).

[22] M. J. Lipp, Y. Kono, Z. Jenei, H. Cynn, and C. Park, J. Phys.: Condens. Matter 25, 345401 (2013).

[23] W. Liu, B. Li, L. Wang, J. Zhang, and Y. Zhao, J. Appl. Phys. 104, 076102 (2008).
[24] P. A. Rigg, C. W. Greeff, M. D. Knudson, G. T. Gray, and R. S. Hixson, J. Appl. Phys. 106, 123532 (2009).

[25] N. Velisavljevic, G. N. Chesnut, L. L. Stevens, and D. M. Dattelbaum, J. Phys.: Condens. Matter 23, 125402 (2011).

[26] Y. Zhao and J. Zhang, Appl. Phys. Lett. 91, 201907 (2007).

[27] J. Zhang, Y. Zhao, P. A. Rigg, R. S. Hixson, and G. T. Gray, J. Phys. Chem. Solids 68, 2297 (2007).

[28] N. V. C. Shekar, K. G. Rajan, and M. S. Division, Bull. Mater. Sci. 24, 1 (2001).

[29] P. A. Rigg, R. A. Saavedra, and R. J. Scharff, J. Phys.: Conf Ser. 500, 032014 (2014).

[30] T. Hattori, H. Saitoh, H. Kaneko, Y. Okajima, K. Aoki, and W. Utsumi, Phys. Rev. Lett. 96, 255504 (2006).

[31] D. W. Dean, R. M. Wentzcovitch, N. Keskar, J. R. Chelikowsky, and N. Binggeli, Phys. Rev. B 61, 3303 (2000).

[32] Y. Kono, C. Park, T. Sakamaki, C. Kenny-Benson, G. Shen, and Y. Wang, Rev. Sci. Instrum. 83, 033905 (2012).

[33] N. Velisavljevic, S. Macleod, and H. Cynn, in Titanium Alloys - Towards Achieving Enhanced Properties for Diversified Applications (InTech Open Source, 2008), Chap. 4.

[34] M. K. Jacobsen and N. Velisavljevic, J. Appl. Phys. 119, 165904 (2016).

[35] P. I. Dorogokupets and A. Dewaele, High Press. Res. 27, 431 (2007).

[36] S. Speziale, C.-S. Zha, T. S. Duffy, R. J. Hemley, and H.-K. Mao, J. Geophys. Res. 106, 515 (2001).

[37] G. Love, C. Koch, H. Whaley, and Z. McNutt, J. Less-Common Met. 20, 73 (1970).

[38] H. Ledbetter, M. Lei, and S. Kim, Phase Transitions 23, 61 (1990).

[39] D. S. Sanditov, M. V. Darmaev, B. D. Sanditov, and V. V. Mantatov, Russ. Phys. J. 52, 386 (2009).

[40] Y. Kono, A. Yamada, Y. Wang, T. Yu, and T. Inoue, Rev. Sci. Instrum. 82, 023906 (2011).

[41] J. Goldak, L. T. Lloyd, and C. S. Barrett, Phys. Rev. 144, 478 (1966).

[42] V. N. Belomestnykh, Tech. Phys. Lett. 30, 91 (2004).

[43] See Supplemental Material at http://link.aps.org/supplemental/ 10.1103/PhysRevB.95.134101 for Results of X-ray Fit Analysis.

[44] E. S. Fisher and C. J. Renken, Phys. Rev. 135, A482 (1964).

[45] E. S. Fisher and C. J. Renken, J. Nucl. Mater. 4, 311 (1961).

[46] H. K. Yeddu, H. Zong, and T. Lookman, Acta Mater. 102, 97 (2016).

[47] A. Heiming, W. Petry, J. Trampenau, M. Alba, C. Herzig, H. R. Schober, and G. Vogl, Phys. Rev. B 43, 10948 (1991).

[48] B. Li, J. Kung, and R. C. Liebermann, Phys. Earth Planet. Inter. 143, 559 (2004).

[49] M. K. Jacobsen, N. Velisavljevic, and S. V. Sinogeikin, J. Appl. Phys. 118, 025902 (2015). 